\title{
Review
}

Journal of Innate

Immunity
J Innate Immun 2012;4:31-40

DOI: $\underline{10.1159 / 000330095}$
Received: April 29, 2011

Accepted after revision: June 16, 2011 Published online: September 6, 2011

\section{S100A8 and S100A9: New Insights into Their Roles in Malignancy}

\author{
Geetha Srikrishna \\ Sanford Children's Health Research Center, Sanford-Burnham Medical Research Institute, La Jolla, Calif., USA
}

\section{Key Words}

S100A8 - S100A9 - Tumor microenvironment · Tumors •

Damage-associated molecular pattern $\cdot$ RAGE .

Myeloid cells

\begin{abstract}
Recent studies have highlighted key roles played by nonneoplastic host cells of the tumor microenvironment, and by secreted factors from tumor and host cells, in promoting malignancy. In this regard, damage-associated molecular pattern (DAMP) molecules such as S100A8 and S100A9, with well-known functions in inflammation, have been increasingly recognized not only as markers, but also as new candidates with important roles in modulating tumor growth and metastasis. This review focuses on our current understanding of the pro- and anti-tumorigenic functions of S100A8 and S100A9. Elucidating molecular pathways mediated by these proteins promises to provide potential novel targets for the development of cancer therapeutics and to establish valid biomarkers to identify early stages of tumor progression.
\end{abstract}

Copyright ๑ 2011 S. Karger AG, Basel

\section{KARGER}

Fax +4161306 1234

E-Mail karger@karger.ch

www.karger.com
(C) 2011 S. Karger AG, Basel

Accessible online at: www.karger.com/jin

\section{Introduction}

Cancers evolve through a complex multi-step process. Decades of research that focused on transformed epithelial cells identified several critical traits which contribute to malignancy: sustained growth signaling, evasion of growth suppression and apoptosis, acquisition of unlimited replication potential, and promotion of angiogenesis, tumor invasion and metastasis [1]. These hallmarks have now been revisited in the light of more recent knowledge on the critical contribution of the tumor microenvironment to malignancy [2]. Tumors are not only made up of the transformed epithelial cells, but they also contain a stromal component of interstitial extracellular matrix, cancer-associated fibroblasts, immune cells, pericytes and endothelial cells that constitute the tumor microenvironment, a dynamic system that actively contributes to the hallmark traits of cancers through cellular, autocrine and paracrine communications [3]. Among the soluble mediators involved in this process, recent studies have brought into focus the role of damage-associated molecular pattern (DAMP) molecules in modulating host immune responses and in promoting tumorigenesis and progression to malignancy $[4,5]$. Although the term DAMP was originally adopted to represent intracellular factors passively released from damaged cells that are rec-
Dr. Geetha Srikrishna

Sanford-Burnham Medical Research Institute

10901, North Torrey Pines Road

La Jolla, CA 92037 (USA)

Tel. +1 858795 5256, E-Mail gsrikrishna@ sanfordburnham.org 
ognized as danger signals by the innate immune system, it has now evolved to include those that are actively secreted from stimulated cells to mediate innate immune responses. This review addresses the contributions of DAMP molecules S100A8 and S100A9 to malignancy, and seeks to complement excellent recent review articles on the subject [6-9], as more novel functions of these proteins in tumors continue to be unraveled.

\section{Structure and Expression of S100A8 and S100A9}

S100A8 and S100A9 belong to a family of 25 homologous low-molecular-weight intracellular calcium-binding proteins that exhibit tissue and cell-specific expression [10-12]. They are characterized by two distinct EF-hand (helix-loop-helix) calcium-binding domains connected by a hinge region. The $\mathrm{N}$-terminal $\mathrm{Ca}^{2+}$ binding domain has lower affinity than the canonical C-terminal domain that allows for functionally important second messenger roles dependent on intracellular $\mathrm{Ca}^{2+}$ levels. Twenty-one of the human S100 genes are clustered at the chromosomal region $1 \mathrm{q} 21$, a region that is frequently deleted, translocated or duplicated in tumors, indicating their possible involvement in malignancy $[10,11]$. Although S100A 8 and S100A9 exist as homodimers similar to many other $\mathrm{S} 100$ proteins, they preferentially form functional anti-parallel heterodimers of S100A8/A9, also known as calprotectin, as well as $\mathrm{Ca}^{2+}$-induced tetramers $[10,11,13]$.

S100A8 and S100A9 are constitutively expressed by myeloid cells, including granulocytes, monocytes, osteoclasts and early-differentiation cells of the myeloid lineage, but not by lymphocytes [12, 14-16]. They comprise $\sim 45 \%$ of neutrophil cytosolic proteins. Deletion of S100A9 gene leads to the absence of S100A8 at the protein level [17]. Phagocyte-specific expression of S100A8 and S100A9 accounts for many of their known functions in the innate immune system. Expression in myeloid cells is down-regulated during differentiation to macrophages and dendritic cells $[14,18]$. In addition, both proteins can be simultaneously induced in monocytes, endothelial cells, keratinocytes or epithelial cells by several mediators, including LPS, TNF $\alpha$, IL1 $\alpha$, IL-1 $\beta$, IL-10 or IL-22 [reviewed in $6,9,16]$. Factors secreted by primary tumors, such as VEGF-A, TGF $\beta$ and TNF $\alpha$ promote induction of S100A8 and S100A9 in macrophages and endothelial cells of premetastatic lungs [19]. Expression is enhanced by stress response modulators such as norepinephrine and by glucocorticoids $[9,16]$. In addition, IL-10, an anti-inflammatory mediator, induces expression of S100A8 and
S100A9 in dendritic cells and potentiates TLR ligand-induced expression of the proteins in macrophages $[9,16]$. This dual induction of expression by both pro- and antiinflammatory mediators is cell-type dependent [16], and suggests that S100A8 and S100A9 can exert opposing roles in inflammation. However, an anti-inflammatory role for the proteins has not been demonstrated in vivo, and therefore molecular mechanisms mediating possible dual roles necessitate further studies.

At the gene level, PU.1 and C/EBP- $\alpha$ and $\beta$ promote transcription of S100A9 in myeloid cells and C/EBP- $\alpha$ and $\beta$ may contribute to expression of both proteins in keratinocytes [9]. Expression in keratinocytes and in hepatocellular tumor cells is also promoted by activation of NF- $\kappa \mathrm{B}$, while AP- 1 appears to negatively regulate expression in keratinocytes $[9,20]$. In addition, STAT-3 promotes transcriptional activation of S100A9 in myeloid progenitors and breast cancer cells $[9,14]$.

\section{Intracellular and Extracellular S100A8 and S100A9}

Like most DAMP molecules, S100A8/A9 lead a double life [21]. In vitro studies indicate that S100A8/A9 contribute to intracellular homeostatic processes that include calcium sensing, activation of NADPH oxidase and arachidonic acid transport [10, 12, 22]. They also play an important role in tubulin-dependent cytoskeletal rearrangements during transendothelial migration of phagocytes $[23,24]$. In addition to intracellular and cell-surface expression, S100A8/A9 have also been detected in the extracellular medium, at times in large amounts. It is the extracellular S100A8/A9 proteins that function as danger signals, promoting immune responses and repair mechanisms during inflammation and, as more recent studies show, in tumors. In fact, S100A8/A9 is a well-known marker of inflammatory conditions such as rheumatoid arthritis, inflammatory bowel disease, multiple sclerosis, cystic fibrosis and psoriasis, and elevated levels have been associated with diabetes, systemic lupus erythematosus, atherosclerosis, vasculitis, hyperzincemia and other inflammatory disorders $[6,12,21]$. It is now well established that they not only serve as markers, but also mediate both anti- and proinflammatory effects [as reviewed in 16]. The proteins, especially S100A8, are susceptible to oxidation by peroxide, hypochlorite and nitric oxide [16]. This susceptibility, combined with their expression in neutrophils and activated macrophages, cells that produce abundant reactive oxygen species during inflammation, indicates that S100A8 and S100A9 may protect tissues against oxi- 
dative damage. However, anti-inflammatory functions of S100A8/A9 have not been demonstrated in vivo. On the other hand, proinflammatory effects of S100A8/A9 have been well established. Leukocyte recruitment is an important function of DAMP proteins. Murine S100A8 exhibits potent chemotactic activity [16], promoting leukocyte recruitment in inflammation, although the chemotactic effects of human S100A8 and S100A9 are less clear. S100A8/ A9 are also important for leukocyte extravasation during inflammation. The availability of S100 $\mathrm{A}^{-/-}$mice, which also lack S100A8, has further helped define important proinflammatory function of S100A8/A9 in vivo. S100A8/ A9 mediate MMP-induced cartilage destruction and chondrocyte death in experimental arthritis [25]. They have also been shown to play critical roles in amplifying endotoxin-induced lethality, and in promoting systemic autoimmunity $[26,27]$.

\section{Secretion of S100A8 and S100A9}

Since S100A8 and S100A9 lack secretion signals, the question arises of how they appear in the extracellular milieu. It is now known that they are released from cells by a Golgi-independent but energy-dependent pathway that requires an intact cytoskeleton and activation of protein kinase C [28]. In this regard, they form part of a growing family of leaderless proteins including growth factors and cytokines that are secreted from cells by unconventional but regulated processes [29]. This growing family consists of IL- $1 \alpha$, IL-1 $\beta$, FGF-1, FGF- 2 and galectins that have predominant extracellular functions, and proteins such as thioredoxin, HMGB1, annexins and S100 proteins that have both intracellular and extracellular functions. Secretion of S100A8/A9 from cells can be both induced and passive. S100A8 and S100A9 are also released during interaction of activated monocytes with $\mathrm{TNF} \alpha$-stimulated endothelial cells [30]. In addition, high levels of S100A8/A9 at inflammatory sites may be due to passive release following neutrophil necrosis during inflammation, since release has been shown to correlate with loss of neutrophil viability [31]. More interestingly, it is now known that S100A8/A9 are also released from neutrophils as part of NETs (neutrophil extracellular traps), which contain chromatin and granule proteins, during a novel form of cell death called NETosis, promoting anti-fungal activity of neutrophils [32]. Extracellular S100A 8 and S100A9 thus represent danger signals that are both actively secreted and passively released from cells by different mechanisms. An important functional aspect of secreted S100 proteins is their ability to act as extracellular DAMP ligands for cell surface receptors, activating signaling cascades and triggering cellular responses, akin to cytokines and chemokines.

\section{S100A8 and S100A9 in Tumors}

The association between inflammation and carcinogenesis has been long recognized [33]. Growing evidence show that chronic inflammation increases the risk of tumorigenesis (inflammation-induced cancer). Even in the absence of underlying inflammation as a causative factor, tumors originating from genetic alterations support the development of a microenvironment composed of immune cells that promote inflammatory responses (cancer-induced inflammation). Increased levels of S100A8/ A9 found in many pathological conditions associated with inflammation therefore predict a possible role in tumorigenesis. In fact, strong up-regulation of these proteins has also observed in many tumors, including gastric, esophageal, colon, pancreatic, bladder, ovarian, thyroid, breast and skin cancers $[6,34]$, contributed both by tumor cells and by infiltrating immune cells in the tumor stroma. Levels of serum S100A8/A9 in tumor-bearing mice was shown to roughly correlate with the quantity of S100A8/A9 secreting myeloid cells in the blood [15]. Tumor cells also produce S100A8/A9 in response to stimuli $[15,20,35]$. For example, phorbol esters stimulate secretion of S100A8/A9 by prostate cancer cells [35] and S100A9 expression is induced in hepatocellular carcinoma through activation of NF- $\mathrm{BB}$ signaling [20]. In addition, S100A8/A9 could also be released by tumor cell necrosis following hypoxia within growing tumors. Regardless of source, it now appears that they play important roles in both inflammation-induced cancer and cancerinduced inflammation, and mediate concentration-dependent anti or pro-tumor responses.

\section{Anti-Tumor Responses}

Evasion of apoptosis is critical for the development and uncontrolled growth of tumors and contributes to overall pathogenesis of malignancy. Tumors acquire resistance to apoptosis through several mechanisms [36]. Several in vitro studies have demonstrated that extracellular S100A8/A9 complex exhibits growth-inhibitory properties and promotes cytotoxicity and apoptosis in many different human and mouse tumor cells [reviewed in 7]. Apoptosis is mediated through a dual mechanism. One is related to zinc exclusion from target cells; suppres- 
sion of intracellular zinc by S100A8/A9 induces cleavage of pro-caspase- 3 . The other is mediated by Bcl 2 family members of the mitochondrial death pathway. S100A8/ A9 decrease the expression of anti-apoptotic proteins $\mathrm{Bcl} 2$ and $\mathrm{Bcl}-\mathrm{X}_{\mathrm{L}}$, and promote mitochondrial release of $\mathrm{OMI} / \mathrm{HtrA} 2$ and Smac/DIABLO, via a process that is independent of RAGE and FADD-dependent death receptors [7]. These studies strongly indicate that S100A8/A9 elicit powerful anti-tumor responses, and that the cell death pathway mediated by these proteins therefore might provide targets for developing novel therapeutic tools against cancers. However, anti-tumor effects of S100A8/A9 have not yet been established in vivo. In contrast, more recent in vitro and in vivo studies indicate that S100A8/A9 mediate several pro-tumor responses. This discrepancy mandates that further studies be required to fully understand any opposing roles in tumorigenesis. With more recent investigations, it is also becoming apparent that the dichotomic effects of S100A8/A9 on tumor cells may in fact be dictated by extracellular concentrations of S100A8/A9, and activation of different signaling pathways.

\section{Anti- or Pro-Tumor Responses May Be Concentration Dependent}

Effective concentrations for promoting apoptosis of tumor cells shown by different studies are in the range of $20-250 \mu \mathrm{g} / \mathrm{ml}$ (approximately 1-10 $\mu \mathrm{M}$ ), while at lower levels $(<20 \mu \mathrm{g} / \mathrm{ml})$ S100A8/A9 seem to promote proliferation of tumor cells $[7,37,38]$. In addition, while the proapoptotic effect of S100A8/A9 appears to be independent of RAGE, effects on tumor proliferation are mediated by RAGE, and involve induction of MAPK signaling and NF- $\kappa$ B activation $[37,38]$. Serum levels of S100A8/A9 in systemic inflammation have been reported to be in the order of $1-6 \mathrm{mg} / \mathrm{ml}$ [8]. However, in tumor-bearing mice or cancer patients, serum levels are in low nanogram to $\mathrm{microgram} / \mathrm{ml}$ levels, although they are higher than seen in sera of healthy controls (reference levels in the low nanogram/ml range) $[15,39,40]$, and unlikely to be high enough to bring about anti-tumor responses seen in vitro. Moreover, based on recent in vivo studies the proteins appear to predominantly mediate pro-tumor responses through autocrine and paracrine mechanisms as discussed below.

Pro-Tumor Responses: Tumor Migration and Invasion

Cancer lethality arises from the intrinsic ability of tumor cells to invade into the surrounding tissue, and to enter blood or lymphatic vessels from where they metastasize into distal organs. Tumor cell invasion and migration are mediated by many coordinated events. S100A8/A9 induce phosphorylation of MAPK in tumor cells and promote activation of NF- $\mathrm{B}[35,37,41]$. Since MAPK activation promotes cell migration, and S100A8 is chemotactic, several studies have examined a possible role of S100A8/A9 in tumor cell migration. S100A8/A9 at $10 \mu \mathrm{g} / \mathrm{ml}$ increase the migration of benign prostate PNT1A cells in a scratch wound healing assay [35], and promote migration of Lewis lung carcinoma cells and B16 melanoma cells in Boyden chambers at very low concentrations $(100 \mathrm{pg} / \mathrm{ml}$ S100A8 and $1 \mathrm{ng} / \mathrm{ml}$ S100A9) [19]. Treatment of B6F10 melanoma cells with S100A8 and S100A9 $(0.2-1 \mu \mathrm{g} / \mathrm{ml})$ stimulates the expression of matrix metalloproteinases associated with tumor metastasis, and promotes chemotactic migration of the cells in a RAGE-dependent manner [42]. At low concentrations $(<2 \mu \mathrm{g} / \mathrm{ml})$ S100A8 and S100A9 also significantly increase migration of SW837 rectal cancer cells, SW480 colon cancer cells and Panc-1 pancreatic cancer cells [43]. In these cells, S100A8 and S100A9 activate signaling through tumor suppressor protein Smad4; transient depletion of $S \operatorname{mad} 4$ using siRNA knockdown reduces S100A8-induced tumor cell migration. These studies indicate that extracellular S100A8 and S100A9 promote tumor cell proliferation and invasion even at low concentrations via activation of intracellular signaling pathways in tumor cells. In addition, intracellular expression of S100A8 and S100A9 by tumors themselves could contribute to tumor invasion. Invasion and migration of SNU484 gastric cancer cells are significantly inhibited when expression of S100A8/A9 by the cells are suppressed by siRNA targeting [44]. S100A8/A9 are highly upregulated in human breast cancer MCF10A cells in a H-Ras specific manner. Induction of S100A8/A9 converts the tumor cells into a migratory phenotype, accompanied by increased expression of matrix metalloproteinases that promote tumor invasion, while siRNA-targeted knockdown of S100A8/A9 expression reduces H-Ras-induced invasion [45]. Tumor cells thus appear to co-opt S100A8/A9-dependent migratory traits of phagocytes for invasion and metastasis.

\section{Pro-Tumor Responses: Activation of Pro-Tumorigenic Genes}

In mouse models of inflammation-associated skin, colon and liver carcinogenesis, S100A8 and S100A9 are strongly upregulated in keratinocytes, myeloid cells and tumor cells, respectively, in response to activation of RAGE and/or NF- $\kappa$ B signaling [20, 38, 46]. In colitis-associated colon carcinogenesis, S100A8- and S100A9-positive cells are present as early responders in inflamed 
mouse colon tissues during initial acute colitis (2 weeks after initiation), but are absent in the low-level chronic inflammation phase observed 6 weeks after induction [38]. However, they reappear in all regions of dysplasia and adenoma 12-20 weeks after disease induction, suggesting that they may have independent roles in the tumorigenic phase [38]. More recently, in an effort to understand molecular mechanisms involved in S100A8/A8mediated tumor-stromal interactions, in vitro and in vivo effects of extracellular S100A8/A9 secreted by myeloid cells in the tumor microenvironment have been further explored. Examination of possible interactions of S100A8/A9 with tumor cells show that they bind to RAGE expressed on tumor cells and promote activation of MAPK signaling pathways and NF- $\kappa B$ activation $[37,38$, 41]. Binding of S100A8/A9 to colon tumor cells is mediated by carboxylated glycans expressed on RAGE $[38,41]$. Since activation of NF- $\kappa \mathrm{B}$-dependent genetic programs in tumor cells and macrophages is critical for development of tumors [47], gene expression studies of tumor cells might provide valuable insight into the consequences of S100A8/A9 activation. In fact, S100A8/A9 activation of colon tumor cells enhances expression of a small cohort of genes, including Cxcl1, Ccl5 and Ccl7, Slc39a10, $L c n 2, Z c 3 h 12 a, E n p p 2$ and other genes, whose products are known to promote leukocyte recruitment, angiogenesis, tumor migration, wound healing, and formation of premetastatic niches in distal metastatic organs [41]. These genes thus represent a distinctive molecular signature of S100A8/A9-activated tumor cells contributing to tumor progression. CXCL1, an S100A8/A9 activated protein, is up-regulated in tumors, and elevated in sera of tumor-bearing wild-type mice, even when tumors are well-contained and before any evidence of metastasis [41]. This suggests that even at low concentrations in the tumor microenvironment or extracellular medium, S100A8/A9 could significantly amplify pro-tumor responses that eventually lead to malignancy. In support of this, mice lacking S100A9 showed a significantly reduced incidence of colon tumors, tumor growth and metastasis, reduced chemokine levels, and reduced infiltration of myeloid cells in different mouse models of colon cancer. Studies using bone marrow chimeric mice show that S100A8/A9 expression on myeloid cells is essential for development of colon tumors [41]. These findings reveal a novel role for myeloid-derived S100A8/A9 in activating specific downstream genes associated with tumorigenesis and in promoting tumor growth and metastasis, and identify them as important players in the molecular communication between tumor and stroma.
Pro-Tumor Responses: Promotion of Premetastatic Niches in Distal Organs

Primary tumors have a tendency to migrate preferentially to specific distal sites. The earliest stages in the metastatic cascade appear to be initiated by permissive niches of hematopoietic cells that form in specific distal organs, which help recruit and support the growth of tumor cells [48]. An important role for S100A8 and S100A9 in the establishment of premetastatic niches in distal organs was first highlighted by the work of Hiratsuka et al. [19]. They demonstrated that soluble factors such as VEGF-A, TGF $\beta$ and $\mathrm{TNF} \alpha$ released by primary tumors induce expression of S100A8 and S100A9 in premetastatic lung endothelium and macrophages. S100A8 and S100A9 secreted by lung stromal cells promote the recruitment of $\mathrm{CD} 1 \mathrm{~b}^{+}$myeloid cells and establishment of premetastatic niches in lungs, to which tumor cells migrate. Treatment of tumor-bearing mice with anti-S100A8 and anti-S100A9 block the migration of myeloid cells and tumor cells to the lung. S100A8 and S100A9 recruit CD11b ${ }^{+}$myeloid cells in the lung through the activation of p38 MAPK, known to promote migration. Subsequently, Hiratsuka et al. [49] showed that S100A8 induced the expression of SAA3 in premetastatic lungs, which stimulated NF- $\mathrm{KB}$ signaling by interaction with TLR4, resulting in accumulation of $\mathrm{CD}_{11} \mathrm{~b}^{+}$myeloid cells. Blocking SAA3 and TLR4 in vivo reduced tumor metastasis, suggesting a paracrine positive feedback in promoting tumor metastasis. Thus, a gradient of S100A8/A9 likely provides a pathway for homing of distal tumor cells to the lungs. In fact, Saha et al. [42] demonstrated the presence of a circulating S100A8/S100A9 gradient from the tail veins to the lungs in uteroglobindeficient mice. Lungs in these mice express high levels of S100A8 and S100A9, to which melanoma cells preferentially migrated. More recently, Connolly et al. [50] showed that formation of premetastatic sites in livers in response to intra-abdominal tumors is also contingent upon the expression of CXCL1, a protein that is now shown to be activated in tumor cells by S100A8/A9 [41]. Again, these studies indicate that tumor cells co-opt chemical cues and migratory gradients used by myeloid cells to metastasize to distal organs, and that S100A8 and S100A9 play an important role in early stages of tumor metastasis.

\section{Pro-Tumor Responses: Accumulation of}

Myeloid-Derived Suppressor Cells

Tumors induce host-driven $\mathrm{T}$ cell tolerance that supports immune evasion, promotes tumor progression, and limits the efficacy of immune-based therapies. Contributing to this immune evasion are myeloid-derived suppres- 
sor cells (MDSC) that have been identified in tumor-bearing mice and in cancer patients [51, 52]. MDSC consist of a heterogenous population of bone-marrow-derived myeloid progenitor cells and immature myeloid cells with potent immunosuppressive effects that promote tumor growth by inhibiting T-cell-driven anti-tumor immune responses through multiple mechanisms [53]. Recent studies show that S100A8 and S100A9 play important roles in the generation and recruitment of MDSC in tumors [14]. S100A8 and S100A9 are downregulated during normal differentiation of myeloid precursors to dendritic cells (DC) and macrophages $[14,18]$. However, as demonstrated by Cheng et al. [14], tumor-derived factors promote sustained STAT3-dependent upregulation of S100A9 in myeloid precursors, which results in inhibition of differentiation to DC and accumulation of MDSC. In support of this, S100A9 $9^{-/}$mice show less accumulation of MDSC, higher rate of tumor rejection, and reduced growth of lymphomas and sarcomas than wild-type controls. On the other hand, overexpression of S100A9 in progenitor cells results in the accumulation of MDSC in tumor-free mice. In a parallel study, Sinha et al. [15] showed that S100A8/A9 are not only expressed by MDSC, but they also have binding sites for S100A8/A9. Part of the binding is mediated by carboxylated glycans and by RAGE, leading to intracellular NF-кB signaling. In addition, tumor cells and MDSC secrete S100A8/A9, and extracellular S100A8/A9 proteins promote MDSC migration. Treatment of tumor-bearing mice with an antibody against carboxylated glycans reduced MDSC recruitment and accumulation in serum and secondary lymphoid organs [15]. These findings strongly indicate that S100A8/A9 support an autocrine feedback loop that sustains accumulation of MDSC in tumors.

Increased numbers of $\mathrm{CD} 1 \mathrm{~b}^{+} \mathrm{Grl}^{+}$cells bearing the classical markers of murine MDSC have been identified in premetastatic lungs and liver in tumor-bearing mice $[41,50,54]$. S100A9-deficient mice show reduced accumulation of $\mathrm{CD} 1 \mathrm{bb}^{+} \mathrm{Grl}^{+}$cells in premetastatic liver and lungs in response to distal colon tumors [41], further substantiating a role for S100A8/A9 in formation of premetastatic niches and accumulation of MDSC.

\section{Receptors That Recognize Extracellular S100A8/A9}

It is now increasingly recognized that extracellular DAMPs mediate autocrine and paracrine signaling through interaction with cell surface pattern recognition receptors such as TLRs and RAGE [5]. By recognizing either pathogen-derived or host-derived danger signals, pattern recognition receptors seem to represent a common pathway to alert the host to danger and to mount intracellular responses.

\section{Toll-Like Receptors}

Toll-like receptors (TLRs) are a family of transmembrane receptors that recognize conserved microbial molecular patterns, and they enable cells of the innate immune system to mount inflammatory responses against pathogens [55]. Using surface plasmon resonance, Vogl et al. [26] provided the first evidence that purified S100A8 specifically bound TLR4-MD2 complex. They also showed that S100A8/A9 amplified endotoxin-mediated inflammatory responses through TLR4. In support of this, bone marrow cells from S100 $\mathrm{A}^{-/-}$mice showed decreased inflammatory response to LPS stimulation, and addition of extracellular S100A8/A9 compensated for this impaired response. In addition, $\mathrm{S} 100 \mathrm{~A} 9^{-/-}$mice were resistant to endotoxin-induced lethal shock and abdominal sepsis. Subsequently, using a mouse autoimmune model, Loser et al. [27] showed that S100A8/A9 locally present in extracellular medium in tissues of a mouse autoimmune model interacted with TLR4. This interaction increased expression of IL-17, which promoted induction of autoreactive CD8+ T cells, and the development of systemic autoimmunity. Also, in an antigen-induced arthritis model, Grevers et al. [56] demonstrated that bone erosions were significantly reduced in S100A9 ${ }^{-/-}$mice. S100A8 appears to promote osteoclast formation and bone resorption in a TLR4-dependent manner, since S100A8-induced effects are blocked in TLR4 ${ }^{-/}$osteoclast precursors. These studies provide compelling evidence for a causal role for S100A8/A9 in inflammatory diseases, through interactions with TLR4.

Recent studies indicate that TLRs also have important roles in tissue repair and carcinogenesis [57]. All TLRs except TLR3 associate with myeloid differentiation factor 88 (MyD88), and this stimulates a kinase cascade resulting in the activation of MAPKs, JNKs, p38 and ERKs, and NF- $\kappa B$ [55]. MyD88 is essential for the promotion of diethylnitrosamine-induced hepatocellular tumors, spontaneous and azoxymethane-induced intestinal tumorigenesis, and chemically induced skin tumors [57]. TLR4 signaling also promotes colitis-induced colon carcinogenesis [58]. However, it is still unclear if S100A8/A9 promote TLR-mediated tissue regeneration and carcinogenesis by direct interaction. In premetastatic lungs of tumor-bearing mice, Hiratsuka et al. [49] identified SAA3 as an important downstream effector of S100A8 and S100A9. SAA3 was shown to be an endogenous ligand for TLR4; 
SAA3 stimulates TLR4 activity and promotes NF- $\mathrm{B}$ activation in hematopoietic cell lines and macrophages and migration of tumor cells, and blocking SAA3 and TLR4 in vivo inhibited tumor metastasis [49]. Therefore, although signaling mediated by a direct interaction of S100A8/A9 with TLR4 is not implicated in early pulmonary metastasis, a paracrine cascade consisting of S100A8/ A9, SAA3 and TLR4 appears to be of critical importance.

\section{Receptor for Advanced Glycation End Products}

RAGE is a type I transmembrane protein, and a signaling receptor of the immunoglobulin superfamily. Although originally identified as a receptor for advanced glycation end products, it is known to bind many structurally unrelated ligands. Numerous studies have implicated RAGE in multiple pathologies, including diabetes, inflammation, neuronal degeneration and cancers, primarily as a receptor and effector of intracellular responses mediated by DAMPs $[59,60]$.

RAGE and S100A8/A9 are co-expressed in tumors and linked to downstream signaling in tumor cells $[35,37,39]$. Recent studies provide more direct evidence for interaction of S100A8/A9 to RAGE on tumor cells [37, 38]. At low concentrations, extracellular S100A8/A9 promote the growth of tumor cells, an effect blocked by RAGE gene silencing or by treatment with anti-RAGE [37]. S100A8/ A9 binds to a small subpopulation of purified RAGE modified by carboxylated glycans [38]. RAGE is also coimmunoprecipitated with S100A8/A9 from colon tumor cells, and S100A8/A9 promote RAGE and carboxylatedglycan-dependent activation of MAPK and NF- $\kappa$ B signaling pathways in colon tumor cells [41]. These findings strongly indicate that the glycosylation status of RAGE dictates responses mediated by some of its ligands. Carboxylated glycans show restricted expression on mouse and human cells of myeloid lineage including monocytes, macrophages and dendritic cells and on endothelial cells. They are absent or undetectable on normal epithelial cells [5]. However, they are expressed on several tumor cells. Inhibiting carboxylated-glycan-dependent interactions using mAbGB3.1, an anti-glycan antibody, colitis-associated colon cancer [38] and accumulation of MDSC in a 4T1 model of metastatic mammary tumor [15].

RAGE ligands are highly elevated during inflammation, and persistent activation of NF- $\kappa \mathrm{B}$ could lead to a positive feedback loop and sustained cellular activation promoting tumor development. In support of this, recent studies indicate a role for RAGE in inflammation-induced carcinogenesis. $\mathrm{RAGE}^{-/}$mice are resistant to the onset of DMBA/TPA-induced skin carcinogenesis and azoxymethane/dextran-sulfate-sodium-induced colon carcinogenesis $[38,46]$. In these models, S100A8/A9 were found to be strongly upregulated in keratinocytes and stromal cells within the tumors. In addition, $\mathrm{RAGE}^{-/-}$ mice show reduced levels of MDSC in the DMBA/TPAinduced skin carcinogenesis implicating RAGE in S100A8/A9-induced MDSC recruitment [46].

Although both TLR4 and RAGE are implicated in S100A8/A9-mediated pathological effects, which receptor and signaling pathways are preferentially employed may depend on the pathological settings, cell types involved and local ligand concentrations. Differential effects of S100A8/A9 could also be mediated by carboxylated glycans, which are expressed on RAGE and not on TLR4. Based on existing studies, it appears that TLR4 plays a more prominent role as a receptor for these ligands on immune cells, contributing to the pathology of sepsis, systemic autoimmunity, rheumatoid arthritis and possibly other inflammatory disorders, while RAGE appears to be a primary mediator of effects of S100A8/A9 on tumor cells. In experimental animal models, blocking carboxylated-glycan-dependent interactions has been more effective than blocking RAGE, suggesting that other carboxylated-glycan-expressing cell surface glycoprotein receptors may also be involved in mediating S100A8/A9 interactions. Specific signaling pathways activated by S100A8/A9 in tumors could also depend on the tumor cell involved. S100A8/A9 induces RAGE- and carboxylatedglycan-dependent phosphorylation of ERK1/ERK2 and SAPK/JNK MAPK in colon tumor cells, but not of p38 [41]. In contrast, in human prostate and breast cancer cells, S100A8/A9 activate p38, but not SAPK/JNK $[35,37]$. p38 and SAPK/JNK MAPK proteins are known to function in cell context and cell type-specific manner to coordinate signaling pathways mediating tumor cell proliferation, survival and migration, and may even exert antagonistic effects, depending on signal duration and cross-talk with other signaling pathways.

\section{Conclusions}

An increasing body of evidence implicates S100A8/A9, present in the tumor microenvironment and external milieu, as danger signals that play important roles in tumor progression through autocrine and paracrine signaling (fig. 1). S100A8 and S100A9 are not only targets of protumorigenic signaling pathways, but they also activate many downstream effector genes in tumor cells that promote tumor progression, all of which serve to amplify a 


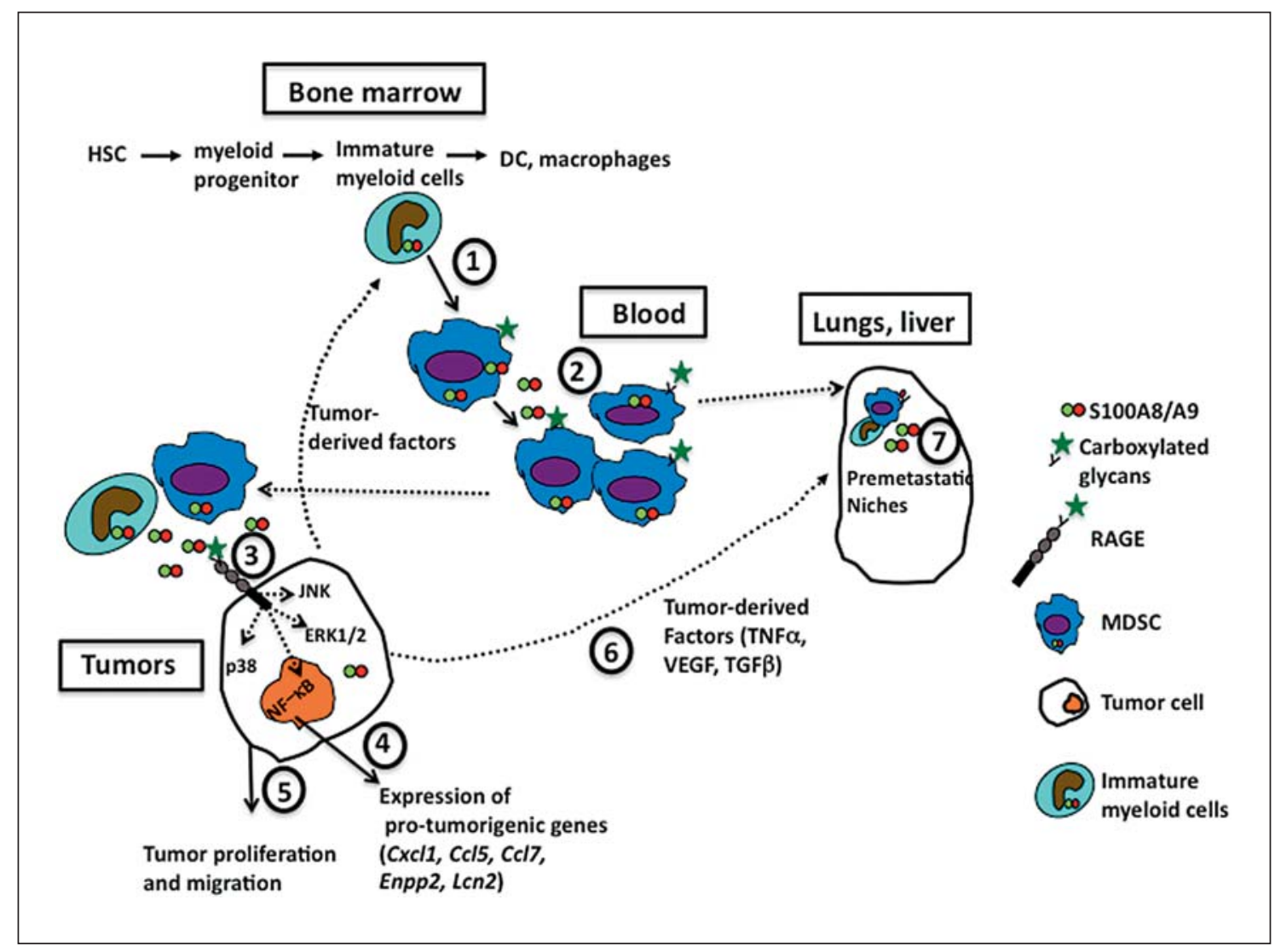

Fig. 1. S100A8/A9 in tumor progression. (1) Tumor-derived factors promote sustained up-regulation of S100A9 in myeloid precursors in the bone marrow, which in turn inhibits normal differentiation of the precursors to dendritic cells and macrophages, and promotes generation of MDSC. (2) MDSC synthesize and secrete S100A8/A9. They also express carboxylated glycans, which provide binding sites for S100A8/A9, promoting activation of intracellular signaling pathways and supporting an autocrine feedback that causes further accumulation of MDSC. (3) MDSC migrate to tumors. At low concentrations, S100A8/A9 secreted by myeloid cells

positive feed-forward signaling during malignancy. S100A8/A9 also exhibit anti-tumorigenic pro-apoptotic effects on tumor cells at high concentrations, even though these effects have yet to be established in vivo. In addition, their expression is downregulated in some tumors, including squamous cell carcinomas of head and neck and of the esophagus. These challenges stipulate that further insights are needed to fully comprehend their role in tumorigenesis. Unraveling molecular pathways mediated by S100A8/A9 would provide exciting opportunities for the study and design of novel cancer therapeutics that selectively target pro- or anti-tumor properties of the proteins, and for the identification of novel biomarkers of cancer. within the tumor microenvironment and by tumor cells themselves bind to RAGE on tumor cells in a carboxylated-glycan-dependent manner, promoting activation of MAPK signaling pathways and NF- $\kappa$ B. (4) Intracellular activation of signaling pathways enhances expression of pro-tumorigenic genes and (5) promotes tumor proliferation and migration. (6) Tumor-derived factors induce expression of S100A8/A9 in distal metastatic organs. (7) They promote formation of premetastatic niches comprising immature myeloid cells, MDSC and endothelial cells, a process dependent on S100A8/A9, SAA3 and TLR4. HSC = Hematopoietic stem cells.

\section{Acknowledgements}

I am grateful to Dr. Hudson Freeze for his long-standing support, and to our many colleagues and collaborators who have made invaluable contributions to our work through the years. Several excellent publications in this field, especially those defining the roles of S100A8 and S100A9 in inflammation, could not be cited due to space constraints. Work from our laboratory on S100A8 and S100A9 in tumors was supported by National Institutes of Health Grant R21-CA127780 (to G.S.). I dedicate this review to the memory of my beloved brother Rajagopalan. 


\section{References}

1 Hanahan D, Weinberg RA: The hallmarks of cancer. Cell 2000;100:57-70.

-2 Hanahan D, Weinberg RA: Hallmarks of cancer: the next generation. Cell 2011;144: 646-674.

3 Tlsty TD, Coussens LM: Tumor stroma and regulation of cancer development. Annu Rev Pathol 2006;1:119-150.

4 Coffelt SB, Scandurro AB: Tumors sound the alarmin(s). Cancer Res 2008;68:6482-6485.

5 Srikrishna G, Freeze HH: Endogenous damage-associated molecular pattern molecules at the crossroads of inflammation and cancer. Neoplasia 2009;11:615-628.

-6 Gebhardt C, Nemeth J, Angel P, Hess J: S100A8 and S100A9 in inflammation and cancer. Biochem Pharmacol 2006;72:16221631.

7 Ghavami S, Chitayat S, Hashemi M, Eshraghi M, Chazin WJ, Halayko AJ, Kerkhoff C: S100A8/A9: a Janus-faced molecule in cancer therapy and tumorgenesis. Eur J Pharmacol 2009;625:73-83.

8 Ehrchen JM, Sunderkotter C, Foell D, Vogl T, Roth J: The endogenous Toll-like receptor 4 agonist S100A8/S100A9 (calprotectin) as innate amplifier of infection, autoimmunity, and cancer. J Leukoc Biol 2009;86:557-566.

$\checkmark 9$ Nemeth J, Angel P, Hess J: Dual role of S100A8 and S100A9 in inflammation-associated cancer. Antiinflamm Antiallergy Agents Med Chem 2009;8:329-336.

10 Donato R: S100: a multigenic family of calcium-modulated proteins of the EF-hand type with intracellular and extracellular functional roles. Int J Biochem Cell Biol 2001;33: 637-668.

-11 Heizmann CW, Fritz G, Schafer BW: S100 proteins: structure, functions and pathology. Front Biosci 2002;7:d1356-d1368.

12 Roth J, Vogl T, Sorg C, Sunderkotter C: Phagocyte-specific S100 proteins: a novel group of proinflammatory molecules. Trends Immunol 2003;24:155-158.

$>13$ Leukert N, Vogl T, Strupat K, Reichelt R, Sorg $\mathrm{C}$, Roth J: Calcium-dependent tetramer formation of S100A8 and S100A9 is essential for biological activity. J Mol Biol 2006;359:961972.

14 Cheng P, Corzo CA, Luetteke N, Yu B, Nagaraj S, Bui MM, Ortiz M, Nacken W, Sorg C, Vogl T, Roth J, Gabrilovich DI: Inhibition of dendritic cell differentiation and accumulation of myeloid-derived suppressor cells in cancer is regulated by S100A9 protein. J Exp Med 2008;205:2235-2249.

- 15 Sinha P, Okoro C, Foell D, Freeze HH, Ostrand-Rosenberg S, Srikrishna G: Proinflammatory S100 proteins regulate the accumulation of myeloid-derived suppressor cells. J Immunol 2008;181:4666-4675.
16 Goyette J, Geczy CL: Inflammation-associated S100 proteins: new mechanisms that regulate function. Amino Acids 2010, DOI: 10.1007/s00726-010-0528-0.

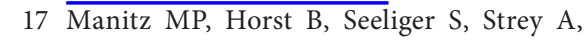
Skryabin BV, Gunzer M, Frings W, Schonlau F, Roth J, Sorg C, Nacken W: Loss of S100A9 (MRP14) results in reduced interleukin-8induced CD11b surface expression, a polarized microfilament system, and diminished responsiveness to chemoattractants in vitro. Mol Cell Biol 2003;23:1034-1043.

18 Lagasse E, Clerc RG: Cloning and expression of two human genes encoding calcium-binding proteins that are regulated during myeloid differentiation. Mol Cell Biol 1988;8: 2402-2410.

19 Hiratsuka S, Watanabe A, Aburatani H, Maru Y: Tumour-mediated upregulation of chemoattractants and recruitment of myeloid cells predetermines lung metastasis. Nat Cell Biol 2006;8:1369-1375.

20 Nemeth J, Stein I, Haag D, Riehl A, Longerich T, Horwitz E, Breuhahn K, Gebhardt C, Schirmacher P, Hahn M, Ben-Neriah Y, Pikarsky E, Angel P, Hess J: S100A8 and S100A9 are novel nuclear factor kappa B target genes during malignant progression of murine and human liver carcinogenesis. Hepatology 2009;50:1251-1262.

21 Foell D, Wittkowski H, Vogl T, Roth J: S100 proteins expressed in phagocytes: a novel group of damage-associated molecular pattern molecules. J Leukoc Biol 2007;81:28-37.

22 Kerkhoff C, Nacken W, Benedyk M, Dagher MC, Sopalla C, Doussiere J: The arachidonic acid-binding protein S100A8/A9 promotes NADPH oxidase activation by interaction with p67phox and Rac-2. FASEB J 2005; 19 : 467-469.

23 Eue I, Pietz B, Storck J, Klempt M, Sorg C: Transendothelial migration of $27 \mathrm{E} 10+\mathrm{hu}-$ man monocytes. Int Immunol 2000;12: 1593-1604.

24 Vogl T, Ludwig S, Goebeler M, Strey A, Thorey IS, Reichelt R, Foell D, Gerke V, Manitz MP, Nacken W, Werner S, Sorg C, Roth J: MRP8 and MRP14 control microtubule reorganization during transendothelial $\mathrm{mi}$ gration of phagocytes. Blood 2004; 104: $4260-4268$

25 van Lent PL, Grevers L, Blom AB, Sloetjes A, Mort JS, Vogl T, Nacken W, van den Berg WB, Roth J: Myeloid-related proteins S100A8/S100A9 regulate joint inflammation and cartilage destruction during antigen-induced arthritis. Ann Rheum Dis 2008;67: $1750-1758$

26 Vogl T, Tenbrock K, Ludwig S, Leukert N, Ehrhardt C, van Zoelen MA, Nacken W, Foell D, van der Poll T, Sorg C, Roth J: Mrp8 and Mrp14 are endogenous activators of Toll-like receptor 4, promoting lethal, endotoxin-induced shock. Nat Med 2007;13: 1042-1049.
27 Loser K, Vogl T, Voskort M, Lueken A, Kupas V, Nacken W, Klenner L, Kuhn A, Foell D, Sorokin L, Luger TA, Roth J, Beissert S: The Toll-like receptor 4 ligands Mrp8 and Mrp14 are crucial in the development of autoreactive CD8+ T cells. Nat Med 2010;16:713-717.

28 Rammes A, Roth J, Goebeler M, Klempt M, Hartmann M, Sorg C: Myeloid-related protein (MRP) 8 and MRP14, calcium-binding proteins of the $\mathrm{S} 100$ family, are secreted by activated monocytes via a novel, tubulin-dependent pathway. J Biol Chem 1997;272: 9496-9502.

-29 Nickel W, Rabouille C: Mechanisms of regulated unconventional protein secretion. Nat Rev Mol Cell Biol 2009;10:148-155.

- 30 Frosch M, Strey A, Vogl T, Wulffraat NM, Kuis W, Sunderkotter C, Harms E, Sorg C, Roth J: Myeloid-related proteins 8 and 14 are specifically secreted during interaction of phagocytes and activated endothelium and are useful markers for monitoring disease activity in pauciarticular-onset juvenile rheumatoid arthritis. Arthritis Rheum 2000; 43:628-637.

31 Voganatsi A, Panyutich A, Miyasaki KT, Murthy RK: Mechanism of extracellular release of human neutrophil calprotectin complex. J Leukoc Biol 2001;70:130-134.

- 32 Urban CF, Ermert D, Schmid M, Abu-Abed U, Goosmann C, Nacken W, Brinkmann V, Jungblut PR, Zychlinsky A: Neutrophil extracellular traps contain calprotectin, a cytosolic protein complex involved in host defense against Candida albicans. PLoS Pathog 2009;5:e1000639.

33 Porta C, Larghi P, Rimoldi M, Totaro MG, Allavena P, Mantovani A, Sica A: Cellular and molecular pathways linking inflammation and cancer. Immunobiology 2009;214: 761-777.

34 Salama I, Malone PS, Mihaimeed F, Jones JL: A review of the $\$ 100$ proteins in cancer. Eur J Surg Oncol 2008;34:357-364.

-35 Hermani A, De Servi B, Medunjanin S, Tessier PA, Mayer D: S100A8 and S100A9 activate MAP kinase and NF-kappaB signaling pathways and trigger translocation of RAGE in human prostate cancer cells. Exp Cell Res 2006;312:184-197.

-36 de Bruin EC, Medema JP: Apoptosis and non-apoptotic deaths in cancer development and treatment response. Cancer Treat Rev 2008;34:737-749.

37 Ghavami S, Rashedi I, Dattilo BM, Eshraghi M, Chazin WJ, Hashemi M, Wesselborg S, Kerkhoff C, Los M: S100A8/A9 at low concentration promotes tumor cell growth via RAGE ligation and MAP kinase-dependent pathway. J Leukoc Biol 2008;83:1484-1492. 
38 Turovskaya O, Foell D, Sinha P, Vogl T, Newlin R, Nayak J, Nguyen M, Olsson A, Nawroth PP, Bierhaus A, Varki N, Kronenberg M, Freeze HH, Srikrishna G: RAGE, carboxylated glycans and S100A8/A9 play essential roles in colitis-associated carcinogenesis. Carcinogenesis 2008;29:2035-2043.

39 Hermani A, Hess J, De Servi B, Medunjanin S, Grobholz R, Trojan L, Angel P, Mayer D: Calcium-binding proteins S100A8 and S100A9 as novel diagnostic markers in human prostate cancer. Clin Cancer Res 2005; 11:5146-5152.

-40 Kim HJ, Kang HJ, Lee H, Lee ST, Yu MH, Kim H, Lee C: Identification of S100A8 and S100A9 as serological markers for colorectal cancer. J Proteome Res 2009;8:1368-1379.

- 41 Ichikawa M, Williams R, Wang L, Vogl T, Srikrishna G: S100A8/A9 activate key genes and pathways in colon tumor progression. Mol Cancer Res 2011;9:133-148.

-42 Saha A, Lee YC, Zhang Z, Chandra G, Su SB, Mukherjee AB: Lack of an endogenous antiinflammatory protein in mice enhances colonization of B16F10 melanoma cells in the lungs. J Biol Chem 2010;285:10822-10831.

-43 Ang CW, Nedjadi T, Sheikh AA, Tweedle EM, Tonack S, Honap S, Jenkins RE, Park BK, Schwarte-Waldhoff I, Khattak I, Azadeh B, Dodson A, Kalirai H, Neoptolemos JP, Rooney PS, Costello E: Smad4 loss is associated with fewer S100A8-positive monocytes in colorectal tumors and attenuated response to S100A8 in colorectal and pancreatic cancer cells. Carcinogenesis 2010;31: 1541-1551.

-44 Yong HY, Moon A: Roles of calcium-binding proteins, S100A8 and S100A9, in invasive phenotype of human gastric cancer cells. Arch Pharm Res 2007;30:75-81
45 Moon A, Yong HY, Song JI, Cukovic D, Salagrama S, Kaplan D, Putt D, Kim H, Dombkowski A, Kim HR: Global gene expression profiling unveils S100A8/A9 as candidate markers in H-ras-mediated human breast epithelial cell invasion. Mol Cancer Res 2008;6:1544-1553.

46 Gebhardt C, Riehl A, Durchdewald M, Nemeth J, Furstenberger G, Muller-Decker K, Enk A, Arnold B, Bierhaus A, Nawroth PP, Hess J, Angel P: RAGE signaling sustains inflammation and promotes tumor development. J Exp Med 2008;205:275-285.

47 Greten FR, Eckmann L, Greten TF, Park JM, Li ZW, Egan LJ, Kagnoff MF, Karin M: IKKbeta links inflammation and tumorigenesis in a mouse model of colitis-associated cancer. Cell 2004;118:285-296.

48 Wels J, Kaplan RN, Rafii S, Lyden D: Migratory neighbors and distant invaders: tumorassociated niche cells. Genes Dev 2008;22: 559-574.

49 Hiratsuka S, Watanabe A, Sakurai Y, AkashiTakamura S, Ishibashi S, Miyake K, Shibuya M, Akira S, Aburatani H, Maru Y: The S100A8-serum amyloid A3-TLR4 paracrine cascade establishes a pre-metastatic phase. Nat Cell Biol 2008;10:1349-1355.

50 Connolly MK, Mallen-St Clair J, Bedrosian AS, Malhotra A, Vera V, Ibrahim J, Henning J, Pachter HL, Bar-Sagi D, Frey AB, Miller G: Distinct populations of metastases-enabling myeloid cells expand in the liver of mice harboring invasive and preinvasive intra-abdominal tumor. J Leukoc Biol 2010;87:713725.

51 Dolcetti L, Marigo I, Mantelli B, Peranzoni E, Zanovello P, Bronte V: Myeloid-derived suppressor cell role in tumor-related inflammation. Cancer Lett 2008;267:216-225.

52 Gabrilovich DI, Nagaraj S: Myeloid-derived suppressor cells as regulators of the immune system. Nat Rev Immunol 2009;9:162-174.
53 Ostrand-Rosenberg S: Myeloid-derived suppressor cells: more mechanisms for inhibiting antitumor immunity. Cancer Immunol Immunother 2010;59:1593-1600.

54 Yan HH, Pickup M, Pang Y, Gorska AE, Li Z, Chytil A, Geng Y, Gray JW, Moses HL, Yang L: Gr-1+CD11b+ myeloid cells tip the balance of immune protection to tumor promotion in the premetastatic lung. Cancer Res 2010;70:6139-6149.

55 Takeda K, Akira S: Toll-like receptors. Curr Protoc Immunol Unit 2007;14:12.

56 Grevers LC, de Vries TJ, Vogl T, AbdollahiRoodsaz S, Sloetjes AW, Leenen PJ, Roth J, Everts V, van den Berg WB, van Lent PL: S100A8 enhances osteoclastic bone resorption in vitro through activation of toll-like receptor 4: implications for bone destruction in arthritis. Arthritis Rheum 2011;63:13651375.

57 Rakoff-Nahoum S, Medzhitov R: Toll-like receptors and cancer. Nat Rev Cancer 2009; 9:57-63.

58 Fukata M, Chen A, Vamadevan AS, Cohen J, Breglio K, Krishnareddy S, Hsu D, Xu R, Harpaz N, Dannenberg AJ, Subbaramaiah K, Cooper HS, Itzkowitz SH, Abreu MT: Tolllike receptor- 4 promotes the development of colitis-associated colorectal tumors. Gastroenterology 2007;133:1869-1881.

59 Logsdon CD, Fuentes MK, Huang EH, Arumugam T: RAGE and RAGE ligands in cancer. Curr Mol Med 2007;7:777-789.

-60 Yan SF, Ramasamy R, Schmidt AM: Receptor for AGE (RAGE) and its ligands-cast into leading roles in diabetes and the inflammatory response. J Mol Med 2009;87:235-247. 